\title{
Técnicas de sutura do tubo digestivo em plano único total, em jejuno de cães: pon- tos de Gambee com nós atados na serosa versus pontos totais atados no lume, so- bre a mucosa ${ }^{1}$
}

\author{
João Luiz Moreira Coutinho Azevedo², Cássio Edvan Paulino da Silva³, Otávio Cansanção Azevedo4, \\ Manoel de Jesus Simões ${ }^{5}$, Ludmila Aimi Kobayashi ${ }^{6}$, Márcio Kozonara ${ }^{6}$
}

\begin{abstract}
Azevedo JL, Silva CEP, Azevedo OC, Simões MJ, Kobayashi LA, Kozonara M. Técnicas de sutura do tubo digestivo em plano único total, em jejuno de cães: pontos de Gambee com nós atados na serosa versus pontos totais atados no lume, sobre a mucosa. Acta Cir Bras [serial online] 2004 Mar-Abr;19(2). Disponível em URL: http://www.scielo.br/acb

RESUMO - Objetivo: Comparar a sutura do tubo digestivo em plano único com pontos totais simples versus pontos especiais de Gambee. Métodos: Foram operados seis cães machos, sem raça definida, pesando entre 7 e $12 \mathrm{~kg}$, anestesiados com pentobarbital sódico a $30 \mathrm{mg} / \mathrm{kg}$. Foi realizada secção transversa do jejuno, a $30 \mathrm{~cm}$ da flexura duodenojejunal, seguida de anastomose término-terminal com a técnica de sutura em plano único total descrita por Gambee, sendo a metade anterior da anastomose suturada com nós atados na superfície serosa e a metade posterior com nós atados sobre a camada mucosa, no lume do órgão. Foram utilizados pontos simples separados de polipropileno monofilamentar azul 4-0, pré-montados em agulha de $2 \mathrm{~cm}$ ( $3 / 8$ de círculo), intervalados de $3 \mathrm{~mm}$. No $7^{\circ}$ dia pósoperatório os animais foram sacrificados e necropsiados. Foram analisadas as aderências na linha de sutura mediante um escore e feita análise macroscópica e microscópica, inclusive histometria. Resultados: As aderências peritoneais se equivaleram em ambas as faces da anastomose. O exame macroscópico da linha de sutura mostrou boa regeneração serosa. Na microscopia observava-se permanência de fenômenos inflamatórios agudos em ambas as suturas. $\mathrm{O}$ realinhamento, a reestruturação e a regeneração das camadas foram adequadas em todo o perímetro da anastomose. Na morfometria, os polimorfonucleares, os macrófagos, os fibroblastos e as fibras colágenas se equivaleram nas duas metades comparadas da anastomose. Conclusão: A reparação do tubo digestivo na região anastomótica em plano único total pela técnica de Gambee se faz de forma similar, quer tenham sido utilizados pontos de sutura especiais de Gambee ou pontos totais simples. Dessa forma, é indiferente a utilização de um ou de outro tipo de sutura aqui estudado, dependendo exclusivamente da preferência do cirurgião.
\end{abstract}

DESCRITORES - Anastomose cirúrgica. Procedimentos cirúrgicos operatórios. Intestino delgado.

\section{Introdução}

Apesar de sempre terem sido considerados relevantes, estudos e pesquisas sobre técnicas de suturas manuais do tubo digestivo estão sendo enfatizados nos últimos anos, principalmente em função da necessidade de se estabelecer qual o método que melhor se adapta à videocirurgia $^{1}$.
As suturas totais gastrintestinais associam viabilidade técnica e segurança. Pontos totais simples e pontos especiais de Gambee são freqüentemente utilizados nessas suturas. Há pertinência em se estabelecer a equivalência ou a diferença na evolução desses dois tipos de pontos de sutura. Gambee ${ }^{2}$ preconizou a sutura em plano único do tubo digestivo mediante pontos totais especiais na face anterior da anastomose, com nós atados no exterior da víscera, na superfície serosa (Figura 1), e pontos totais simples na face posterior, com nós atados no lume do órgão, sobre a camada mucosa (Figura 2). Seguiram-se estudos clínicos ${ }^{2,3,4,5,6,7}$ e experimentais ${ }^{8,9,10,11}$ com essa técnica.

\footnotetext{
1. Trabalho realizado no Laboratório da Disciplina de Técnica Operatória e Cirurgia Experimental do Departamento de Cirurgia da Universidade Federal de São Paulo - Escola Paulista de Medicina (UNIFESP-EPM).

2. Professor Adjunto do Departamento de Cirurgia da UNIFESP-EPM.

3. Pós-Graduando do Programa de Cirurgia e Experimentação da UNIFESP-EPM.

4. Residente de Gastroenterologia Cirúrgica do Hospital do Servidor Público do Estado de São Paulo.

5. Professor Livre Docente do Departamento de Morfologia da UNIFESP-EPM.

6. Alunos do Curso de Graduação em Medicina da UNIFESP-EPM.
} 


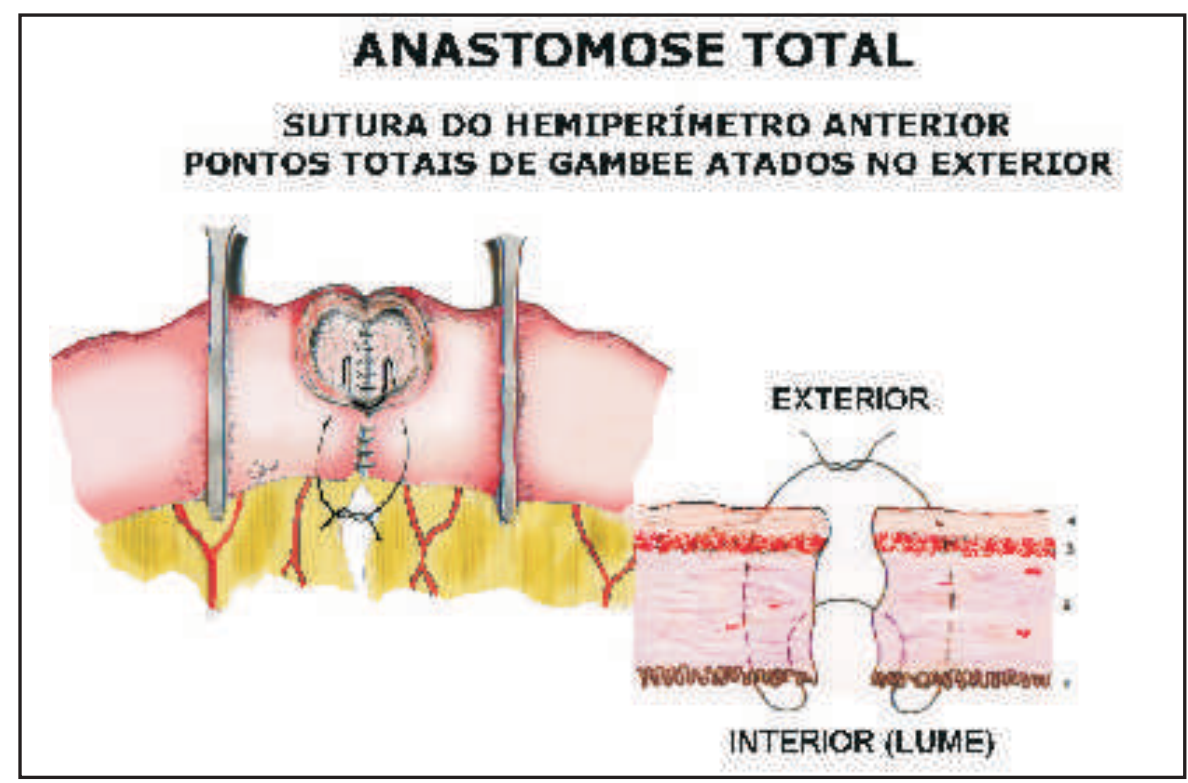

FIGURA 1 - Pontos especiais de Gambee (parede anterior) Inicia-se a execução do ponto de fora para dentro do intestino, mediante a perfuração das camadas serosa (4), muscular externa (3), submucosa (2) e mucosa (1), até o lume intestinal .A seguir, a agulha executa um movimento de dentro para fora, ultrapassando apenas a camada mucosa (1) e saindo do segmento intestinal entre as camadas mucosa (1 e submucosa (2), em direção ao segmento intestinal oposto, no qual penetra entre a mucosa (1) e a submucosa (2), dirigindo-se para o lume do órgão. Em continuação, a agulha ultrapassa todas as camadas intestinais, de dentro para fora, atando-se os nós na superfície serosa (4).

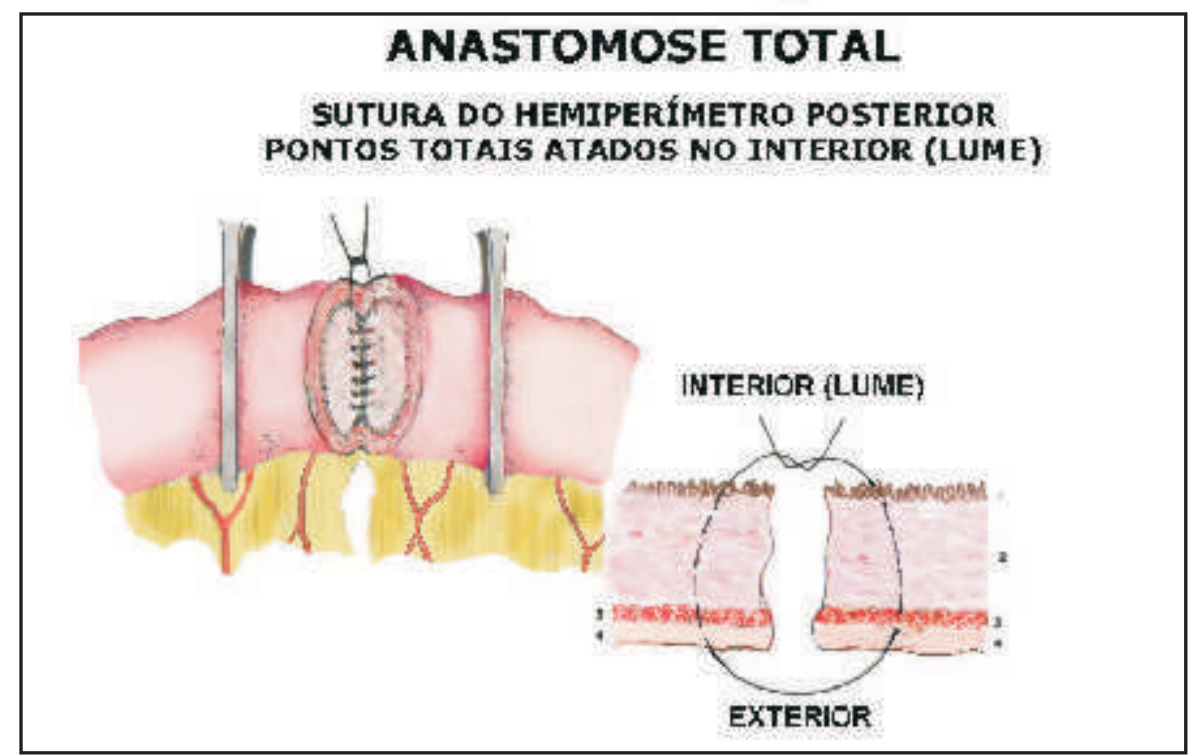

FIGURA 2 - Ponto simples total (parede posterior) Inicia-se a execução do ponto de dentro para fora de um dos cotos intestinais, mediante a perfuração das camadas mucosa (1), submucosa (2), muscular (3) e serosa (4), até o exterior do intestino. A seguir, a agulha executa um movimento de fora para dentro do coto intestinal oposto, transfixando todas as camadas e emergindo no lume do órgão. Os nós são atados na superfície da camada mucosa.

Há trabalhos experimentais nos quais foram utilizados, em toda o perímetro da anastomose, pontos totais simples atados no lume ${ }^{7,12,13}$. Em outras pesquisas foram utilizados apenas pontos totais simples atados na superfície serosa ${ }^{14}$.

Ainda há pesquisas experimentais ${ }^{15,18} \mathrm{e}$ clínicas ${ }^{19}$ com pontos totais especiais de digestivo (uma total e outra parcial - Figura 1) em cada coto a ser anastomosado, e os pontos totais da parede posterior (com nós atados no lume, sobre a mucosa), transfixa apenas uma vez cada coto (Figura 2), a reparação resultante pode diferir. Para responder a esta questão, não pôde ser encontrada pesquisa alguma comparando a evolução do reparo de víscera suturada com pontos totais atados no lume versus pontos especiais de Gambee atados no exterior.

O objetivo deste trabalho é estudar comparativamente a reparação de segmento do tubo digestivo suturado com pontos totais simples atados no lume do órgão, sobre a mucosa versus a reparação com pontos totais especiais de Gambee atados no exterior, sobre a serosa.

\section{Métodos}

Foram operados seis cães machos, sem raça definida, pesando entre 7 e $12 \mathrm{~kg}$, procedentes do Canil da Universidade Federal de São Paulo, Escola Paulista de Medicina, anestesiados com pentobarbital sódico a $30 \mathrm{mg} / \mathrm{kg}$. Foi realizada secção transversa do jejuno, a $30 \mathrm{~cm}$ da flexura duodenojejunal, seguida de anastomose términoterminal com a técnica de sutura em plano único total descrita por Gambee ${ }^{1}$ (Figura 3 ), sendo a metade anterior da anastomose suturada com nós atados na superfície serosa (Figura 1) e a metade posterior com nós atados sobre a camada mucosa, no lume do órgão (Figura 2). Foram utilizados pontos simples separados de polipropileno monofilamentar azul 4-0, pré-montado em agulha de $2 \mathrm{~cm}$ (3/8 de círculo), intervalados de $3 \mathrm{~mm}$. No $7^{\circ}$. dia pós-operatório os animais foram sacrificados e necropsiados. Aderências na linha de sutura foram avaliadas mediante um escore (Figura 4), no qual foi atribuído um ponto por cada quarta parte da extensão da linha de sutura do hemiperímetro anterior ou posterior comprometido com aderências. Na microscopia (H-E e Masson) a inflamação, a cicatrização e a regeneração das camadas foram observadas, e foram contados neutrófilos, macrófagos, fibroblastos e fibras colágenas. O estudo morfométrico (histometria) foi realizado com a utilização de ocular integradora "Intergrationplatte II" de 10 aumentos, fabricação Zeiss, contendo retículo de 25 pontos de interseção das linhas, acoplada a uma objetiva Zeiss 
de 100 aumentos, com aumento final de 1000 diâmetros. Para a contagem diferencial das estruturas observadas nos pontos de interseção das linhas do retículo ("hits"), foram considerados quatro padrões morfológicos (elementos), sendo três deles constituídos por células e o quarto por substância intercelular, a saber: células polimorfonucleadas (neutrófilos); células mononucleadas arredondadas ou discretamente ovóides (macrófagos); células mononucleadas acentua- damente ovóides ou fusiformes (fibroblastos); condensações bem individualizadas de estruturas fibrilares (fibras colágenas). O retículo projetado permitiu a contagem dos elementos nos "hits", ou seja, dos quatro padrões morfológicos citados que se situavam nos pontos de interseção das linhas dos retículos. Na circunstância da estrutura situada no "hit" não corresponder a nenhum dos quatro padrões morfológicos citados, nenhum registro era feito. Dessa forma, nas preparações oriundas de cada animal (Figura 5) foram examinados, em cinco campos microscópicos, os elementos que incidiam em cada um dos 25 retículos (125 retículos examinados por animal), sendo registrado o número de vezes que cada um dos padrões morfológicos descritos incidiu nos retículos. A seguir foi obtida a média aritmética. Foi utilizada a prova de Wilcoxon para duas amostras relacionadas na avaliação das aderências peritoneais e dos resultados morfométricos.

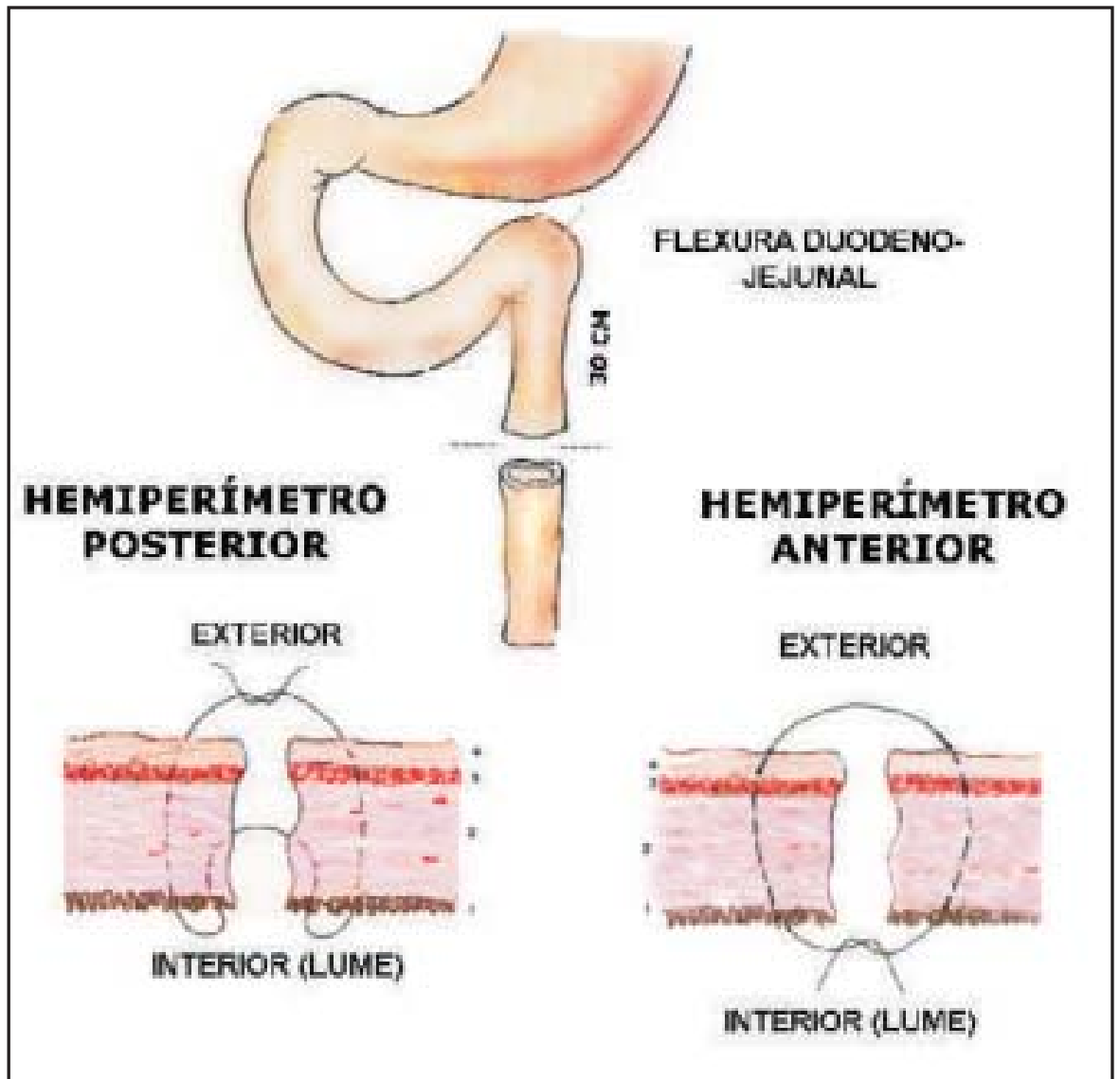

FIGURA 3 - Secção transversa do jejuno, a $30 \mathrm{~cm}$ da flexura duodenojejunal, seguida de anastomose término-terminal com a técnica de sutura em plano único total descrita por Gambee, sendo a metade anterior da anastomose suturada com nós atados na superfície serosa (Figura 1) e a metade posterior com nós atados sobre a camada mucosa, no lume do órgão (Figura 2).

\section{Resultados}

Nenhuma complicação pós-operatória ocorreu. À necrópsia não foram evidenciadas anormalidades. As aderências peritoneais se equivaleram em ambas as faces da anastomose (Tabela 1). Ao exame macroscópico da linha de sutura notavase hipermia da mucosa (Figura 6), com boa regeneração serosa. $\mathrm{Na}$ microscopia observava-se permanência de fenômenos inflamatórios agudos tanto na face anterior (pontos atados na serosa) quanto na posterior da anastomose (pontos atados no lume), com a presença de edema, neutrófilos e microabscessos (Figura 7). Havia granulomas de corpo estranho do tipo imunologicamente ativos em torno dos fios de sutura (Figura 8).
O realinhamento, a reestruturação e a regeneração das camadas foram adequados em todo o perímetro da anastomose.

Na morfometria, os polimorfonucleares, os macrófagos, os fibroblastos e as fibras colágenas se equivaleram nas duas metades comparadas da anastomose (Tabela 2). 


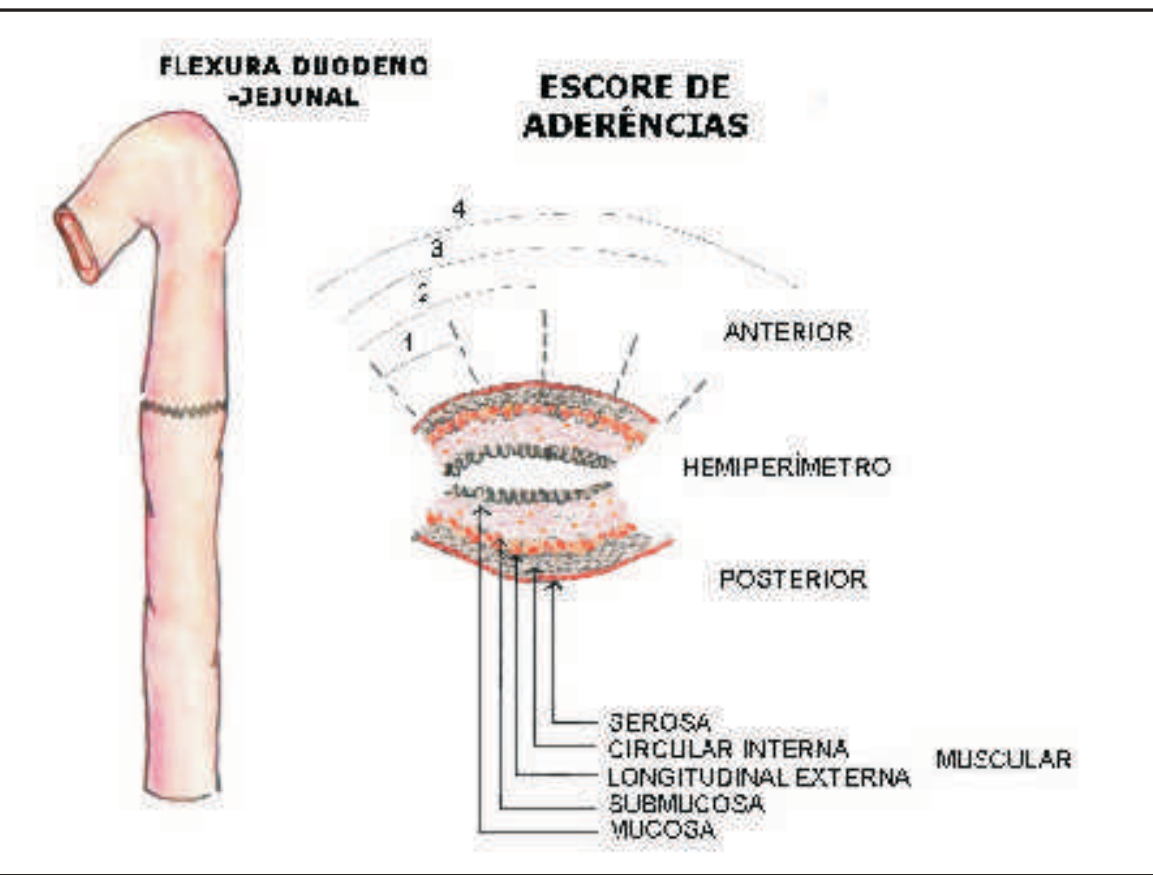

FIGURA 4 - Escore de aderências ao peritônio no qual foi atribuído um ponto por cada quarta parte da extensão da linha de sutura do hemiperímetro anterior ou posterior comprometido.

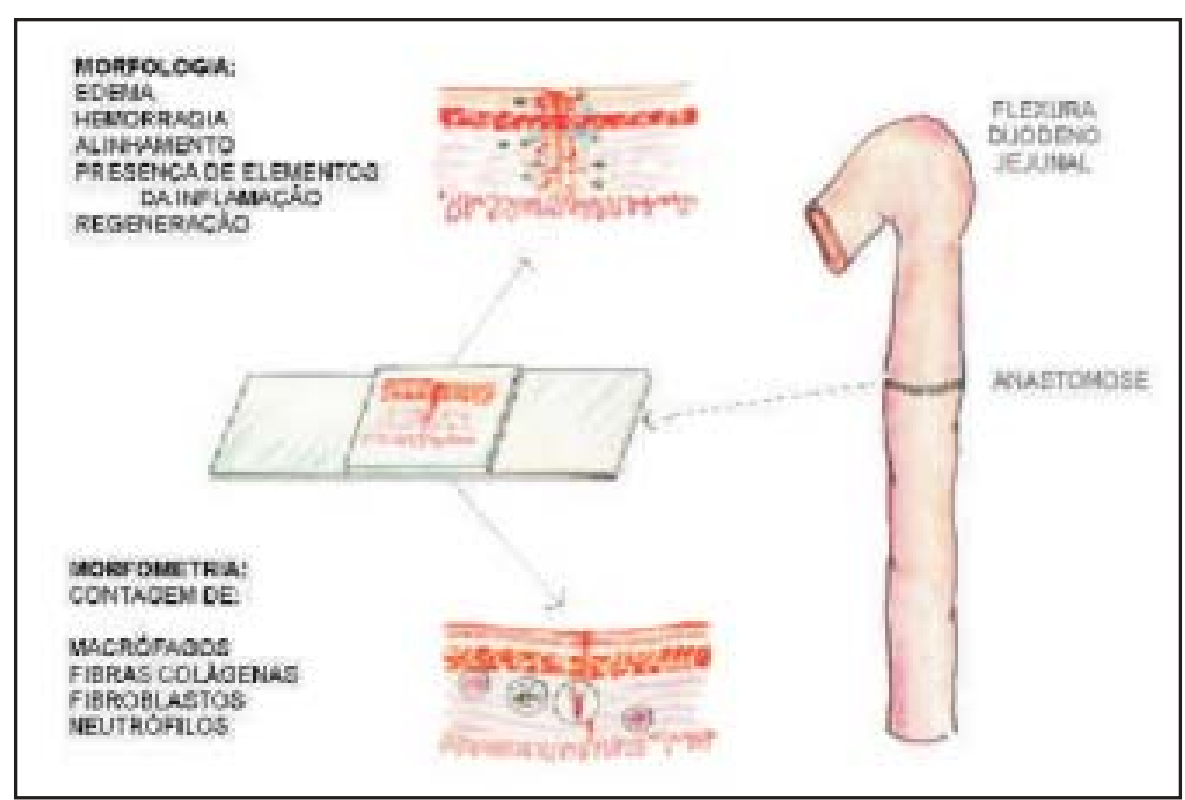

FIGURA 5 - Preparações oriundas de cada animal em cinco campos microscópicos (H-E e Masson).

TABELA 1 - Escore das aderências peritoneais. Pela prova de Wilcoxon: SA = SP

\begin{tabular}{cc}
\hline Sutura total anterior (SA) & Sutura total posterior (SP) \\
\hline 2 & 0 \\
1 & 1 \\
0 & 0 \\
0 & 0 \\
0 & 2 \\
0 & 0 \\
$\mu=0,5$ & $\mu=0,5$ \\
\hline
\end{tabular}




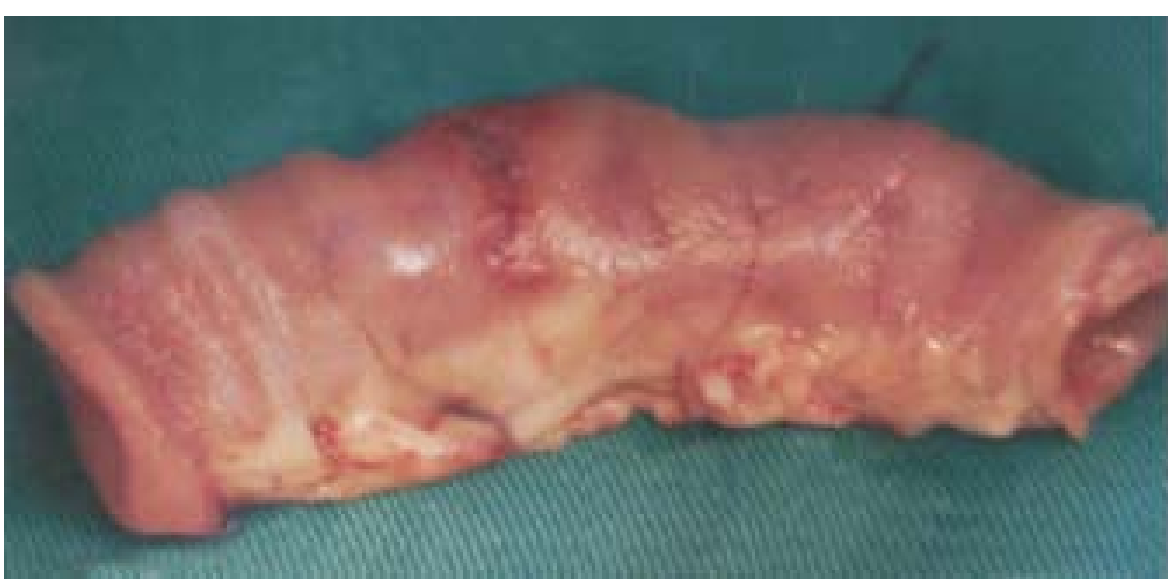

FIGURA 6 - Exame macroscópico da linha de sutura: nota-se hipermia e boa regeneração serosa.

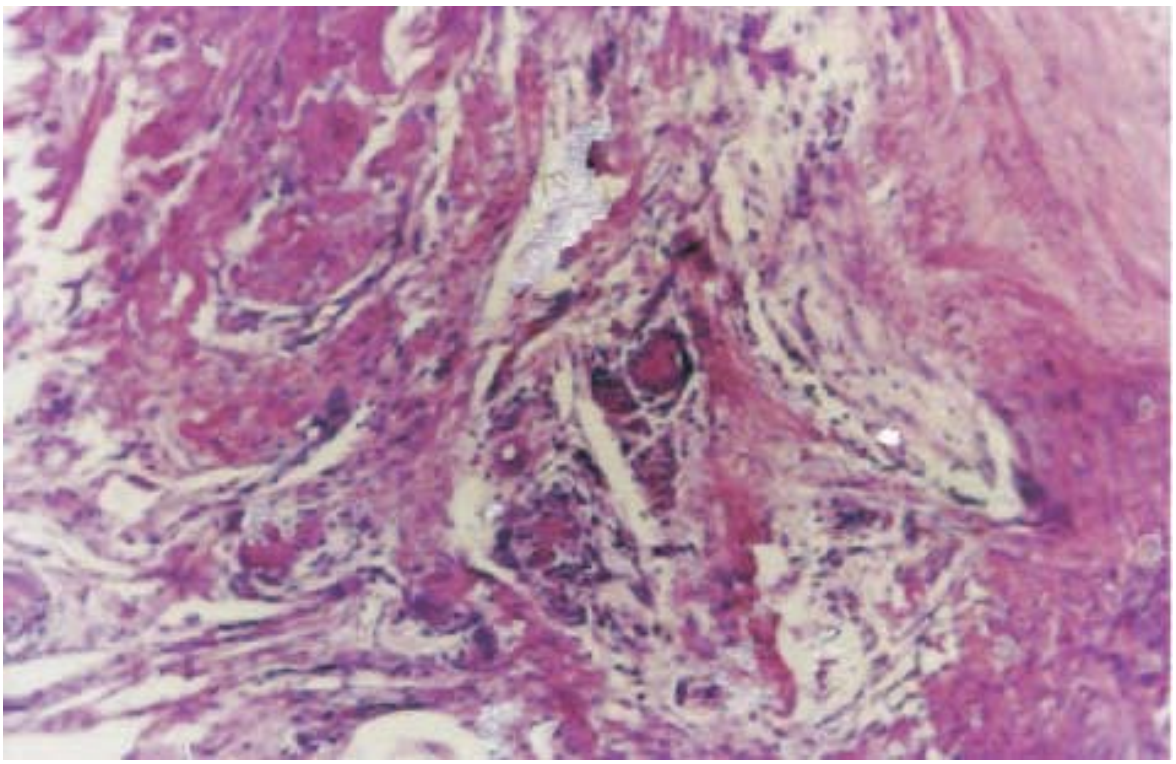

FIGURA 7 - Fotomicrografia $(100 \mathrm{x})$ em zona de anastomose de parede jejunal suturada com pontos totais de Gambee. Observa-se permanência de fenômenos inflamatórios agudos.

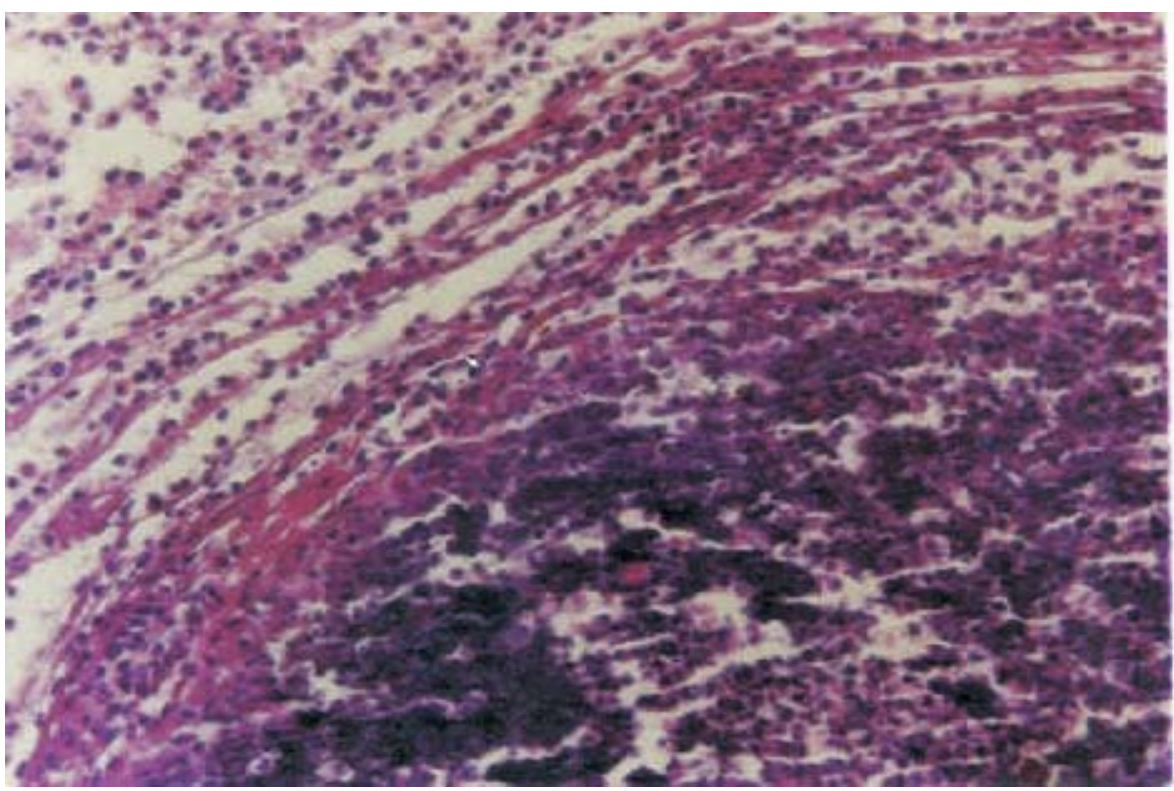

FIGURA 8 - Fotomicrografia $(100 \mathrm{x})$ em zona de anastomose de parede jejunal suturada com pontos totais de Gambee. Observe-se granulomas de corpo estranho do tipo imunologicamente ativos em torno dos fios de sutura.

\section{Discussão}

Nesta pesquisa, tratou-se de verificar a eventual existência de diferenças entre o reparo do intestino suturado com pontos de Gambee, atados na serosa, e com pontos simples totais atados no interior do lume da víscera, sobre a mucosa. Esse particular aspecto nunca havia sido antes investigado, ao que parece.

A boa evolução clínica dos animais e a ausência de anormalidades à necrópsia confirmaram a adequação da anastomose em plano único total com a técnica descrita por Gambee ${ }^{2}$.

À microscopia, a permanência de focos de inflamação aguda no $7^{\circ}$ dia pós-operatório - verificada nesta pesquisa em ambas as faces da anastomose (Figura 7) é devida ao envolvimento da camada mucosa na linha de sutura ${ }^{17,18}$, com focos de necrose avascular (isquêmica) dessa camada, determinados pelo angustiamento tecidual dos pontos de sutura, e não depende do tipo de ponto total.

O tipo de inflamação granulomatosa exuberante em torno do fio de popipropileno utilizado na sutura, encontrada nesta pesquisa, em contraste com os granulomas de corpo estranho inertes observados em outros trabalhos ${ }^{23,24,25,26}$, com o mesmo tipo de fio de sutura, provavelmente se deveu, aqui, à presença de bactérias oriundas do lume intestinal no tecido desvitalizado aderido ao fio. Esse tecido é resultante da necrose isquêmica da mucosa determinada pelo angustiamento tecidual, próprio do ponto cirúrgico total. A contaminação do fio de sutura por bactérias fecais transforma o fio de inerte (material inorgânico sintético estéril) em imunologicamente reativo, porque as bactérias que colonizam o tecido necrótico aderido a ele evocam um granuloma de corpo estranho imunologicamente ativo, numa reação do hospedeiro às proteínas estranhas dessas bactérias.

A inflamação aguda e crônica é passível de ser quantificada (Tabela 2) por histometria ${ }^{23,24,25,26,27}$, a qual, nesta pesquisa, demonstrou a similaridade do reparo intestinal sob os dois tipos de pontos de sutura. Aderências peritoneais também se equivaleram.

Portanto, a reparação do tubo digestivo na região anastomótica em plano único total pela técnica de Gambee se faz de 
forma similar, quer tenham sido utilizados pontos de sutura especiais (com nós atados na serosa) ou pontos totais simples (com nós atados no lume, sobre a mucosa).
Dessa forma, é indiferente a utilização de um ou de outro tipo de sutura aqui estudado, dependendo exclusivamente da preferência do cirurgião.
TABELA 2 - Histometria de macrófagos, fibroblastos, fibras colágenas e neutrófilos por campo microscópico (média de 5 campos). Pela prova de Wilcoxon: sutura anterior $(\mathrm{SA})=$ sutura posterior $(\mathrm{SP})$ para todos os elementos analisados.

\begin{tabular}{cccccccc}
\hline \multicolumn{2}{c}{ Macrófago } & \multicolumn{2}{c}{ Fibroblastos } & \multicolumn{2}{c}{ Fibras Colágenas } & \multicolumn{2}{c}{ Neutrófilos } \\
SA & SP & SA & SP & SA & SP & SA & SP \\
9 & 8 & 7 & 11 & 43 & 39 & 11 & 14 \\
6 & 7 & 12 & 9 & 39 & 45 & 13 & 12 \\
5 & 8 & 16 & 10 & 38 & 41 & 10 & 11 \\
9 & 3 & 8 & 9 & 44 & 29 & 9 & 8 \\
5 & 6 & 13 & 15 & 45 & 39 & 13 & 13 \\
4 & 5 & 12 & 9 & 41 & 44 & 14 & 13 \\
$\mu=6,1$ & $\mu=6,1$ & $\mu=11,3$ & $\mu=10,5$ & $\mu=41,6$ & $\mu=39,5$ & $\mu=11,6 \quad \mu=11,6$ \\
\hline
\end{tabular}

\section{Referências}

1. Waninger J, Salm R, Imdhal A, Haberstroh J, Schoop C, Voshege M, Farthmann EH. Comparison of laparoscopic handsewn suture techniques for experimental smallbowel anastomoses. Surg Laparosc Endosc 1996;6:282-9.

2. Gambee LP. A single layer open intestinal anastomosis applicable to the small as well as the large intestine. West J Surg 1951;59:1-5.

3. Azevedo JLMC. Experiência clínica com a sutura de Gambee. In: Congresso Brasileiro de Medicina Militar, 5, Rio de Janeiro, 1972c. Temas Livres.

4. Azevedo JLMC, Fujimura I, Boarini P, Scomparini CE. Análise da evolução pósoperatória de pacientes submetidos a anastomoses do tubo digestivo em plano único de sutura. In: Congresso Brasileiro de Cirurgia, 20, Rio de Janeiro, 25 a 29 de julho de 1993. Temas Livres.

5. Everett WG. A comparison of one layer and two layer techniques for colorectal anastomosis. Br J Surg 1975;62:135-40.

6. Gambee LP, Garnjobst W, Hardwick CE. Ten years' experience with a single layer anastomosis in colon surgery. Am J Surg 1956;92:222-7.

7. Hamilton JE. Reappraisal of open intestinal anastomoses. Ann Surg 1967;165:917-24.

8. Azevedo JLMC. Estudo comparativo de métodos de suturas intestinais: intestino delgado de cães. In: Congresso Brasileiro de Medicina Militar, 5, Rio de Janeiro, 1972a. Temas Livres.

9. Azevedo JLMC. Estudo comparativo de métodos de suturas intestinais: intestino grosso de coelhos. In: Congresso Brasileiro de Medicina Militar, 5, Rio de Janeiro, 1972b. Temas Livres.

10. Azevedo JLMC, Simões MJ, Stavale JN, Novo NF, Juliano Y. Avaliação da cicatrização e da integridade do colo anastomosado após rotação, em coelhos. Rev Col Bras Cir 1992;19:120-5.

http://www.cirurgiaonline.med.br/artigos/ rcbc1992-5/

11. McAdams AJ, Meikle AG, Taylor JO. One layer or two layer intestinal anastomoses? Am J Surg 1970;120:546-50.

12. Carril CF. Anastomoses intestinais: métodos invaginantes e métodos por aposição em mesmo alinhamento: estudo experimental comparativo com apresentação de um processo original. São Paulo, 1970. (Tese Docência Livre - Faculdade de Medicina de Ribeiro Preto da Universidade de São Paulo)

13. Guimares AS, Ferreira AL, Aprili F, Carril CF. Comparação entre anastomoses intestinais em 1 e 2 planos de sutura mediante estudo da rede vascular sanguínea e da cicatrização: trabalho experimental no intestino delgado do cão. Rev Ass Med Bras 1973;20:97-101.

14. Letwin E, Williams HTG. Healing of intestinal anastomosis. Can J Surg 1967;10:109-16.

15. Abramowitz HB, McAlister WH. A comparative study of small-bowel anastomoses by angiography and microangiography. Surgery 1969;66:564-9.

16. Abramowitz HB, Butcher HR. Everting and inverting anastomoses: an experimental study of comparative safety. Am J Surg 1971;121:52-6.

17. Azevedo JLMC. Estudo comparativo entre as anastomoses em plano único extramucoso e total, em colo de coelhos. São Paulo, 1988. 108p. (Tese - Mestrado Escola Paulista de Medicina).

18. Azevedo JLMC, Goldenberg S, Simões MJ, Stavale, JN. Estudo comparativo entre as anastomoses em plano único extramucoso e total, em colo de coelhos. Acta Cir Bras 1990;5:6-12.

http://www.cirurgiaonline.med.br/artigos/ acb1990-1/
19. Weinberg JA. Vagotomy and piloroplasty in the treatment of duodenal ulcer. Am J Surg 1963;105:347-51.

20. Hardy KJ. Suture anastomosis: an experimental study using limited suturing of the small bowel in the dog. Arch Surg 1968;97:586-9.

21. Mantovani M, Leonardi LS, Alcântara FG, Medeiros RR. Estudo comparativo entre diferentes variedades de sutura em um e dois planos no intestino delgado. Trabalho experimental no cão. Rev Ass Med Bras 1976;22:79-86.

22. Rosenberg D, Frazatto Jr C, Mantovani M, Vieira RW, Medeiros RR. Estudo experimental comparativo entre as suturas em plano único total, extramucoso e de eversão. Rev Paul Med 1973;81:5-12.

23. Azevedo JLMC. Efeitos da ofloxacina na inflamação induzida em ratos. São Paulo, 1991. 164p. (Tese - Doutorado - Escola Paulista de Medicina)

24. Azevedo JLMC, Milanez M, Kosmiskas J, Lee C, Rondina F, Nogueira M, Meziara H, Bancher M. Proposta de modelo experimental (ratos) constituído por granuloma induzido por corpo estranho inerte: subsídios para o estudo da reação tecidual aos materiais implantados e da ação antiproliferativa de drogas sobre células inflamatórias. In: Congresso de Técnica Cirúrgica e Cirurgia Experimental, Belo Horizonte, 24 a 27 de maio de 1992. Temas Livres.

25. Azevedo JLMC, Plapler H, Ortiz V, Lapa AJ, Emin JAS, Simões MJ, Juliano NF, Santos P, Ferreira MHC. Efeitos da ofloxacina no granuloma de corpo estranho induzido por fio de sutura (prolene), em ratos. Rev Bras Cir 1992;82:189-95.

http://cirurgiaonline.med.br/artigos/rpc1992.

26. Azevedo JLMC, Kosmiskas J, Lee C, Rondina F, Nogueira M, Meziara H, Bancher M, Milanez M. Efeitos da indometacina no granuloma induzido por fio de polipropileno, em ratos. In: Congresso De Técnica Cirúrgica E Cirurgia Experimental, 2, Belo Horizonte, 24 a 27 de maio de 1992.

27. Azevedo JLMC, Simões MJ, Santos P, Novo NF. Anastomoses em plano único extramucoso versus anastomoses em plano único total, em jejuno de cães. In: Congresso Brasileiro De Cirurgia, 20, Rio de Janeiro, 25 a 29 de julho de 1993. Temas Livres.

28. Azevedo JLMC, Azevedo GC, Azevedo O, Santos P, Togni F, Izecksonh S. Técnicas de sutura do tubo digestivo em plano único extramucoso, em jejuno de cães: pontos atados na superfície serosa versus pontos atados no lume, sobre a submucosa. Rev Bras Cir 1997; 87:119-26. http://www.cirurgiaonline.med.br/artigos/ rbc1997-3/

29. Azevedo JLMC, Azevedo O, Azevedo G, Santos P, Ricci M, França MS. Suturas do tubo digestivo em plano único: comparação entre as principais técnicas, em cães. Rev Col Bras Cir 1995;22:205-11. http:// www.cirurgiaonline.med.br/artigos/ rcbc_ano4-6/ 


\begin{abstract}
Azevedo JL, Silva CEP, Azevedo OC, Simões MJ, Kobayashi LA, Kozonara M. Single layer anastomosis of digestive tract with thru-and-thru sutures, in dogs: Gambee's stitch knotted under viscous surface, on serosa, versus simple stitch knotted inside the lumen, under mucosa. Acta Cir Bras [serial online] 2004 Mar-Apr;19(2). Available from URL: http://www.scielo.br/acb

ABSTRACT - Purpose: To compare two types of single layer thru-and-thru sutures of the digestive tract: Gambee's stitches with knots fastened in the serosal surface versus simple stitches knotted in the intestinal lumen, under the mucosa. Methods: Six male mongrel dogs were operated weighing between 7 and $12 \mathrm{~kg}$, anesthetized with sodic pentobarbital to $30 \mathrm{mg} / \mathrm{kg}$. Transverse section of the jejunum was accomplished, to $30 \mathrm{~cm}$ of the angle of Treitz, followed by end-to-end anastomosis with interrupted single layer Gambee's stitches fastened in the serosal surface in the anterior half-part of the anastomosis and simple stitches knotted in the viscous lumen, under the mucosal layer, in the posterior half-part of the anastomosis. After slaughter at the $7^{\text {th }}$ postoperative day the peritoneal adhesions were analyzed in the suture line by a score and macroscopic and microscopic analysis were made. Results: Peritoneal adhesions were equivalent in both types of sutures. The intestinal loop showed good serosal regeneration at the suture line. Under microscopy view, residuals acute inflammatory phenomena remain in both sutures. Sparse focus of ischemic necrosis of the mucosa was observed along the suture line, in both types of sutures. Realignment and regeneration of the layers were present along the entire perimeter of the anastomose. In the morphometric analysis, the neutrophils, the macrophages, the fibroblasts and the collagenous fibers were equivalent in number in the two compared half-perimeters of the anastomose. Conclusion: The repair of the digestive tract in the anastomotic area after single layer sutures by the technique of Gambee is made in a similar way, despite of special Gambee's stitches or simple total sutures have been used. In that way, it is indifferent the use of both suture type here studied, depending exclusively on the surgeon's preference.
\end{abstract}

KEY WORDS - Surgical anastomosis. Operative surgical procedures. Small intestine.

Conflito de interesse: nenhum Fonte de financiamento: nenhuma

Correspondência

João Luiz Moreira Coutinho Azevedo

Rua Botucatu, 740 - Edificio da Cirurgia Experimental

04023-062 São Paulo - SP

jozevedo.dcir@epm.br

www.cirurgiaonline.med.br

Data do recebimento: 03/01/2004

Data da revisão: 26/01/2004

Data da aprovação: 12/02/2004

*Fotos coloridas disponíveis em www.scielo.br/acb 